\title{
Síndrome de Ramsay-Hunt en Pediatría: Reporte de cuatro casos y revisión de la literatura
}

\author{
Carmen Sandoval C., Alicia Núñez F., Macarena Lizama C., Cynthia Margarit S., Katia Abarca V. y Raúl Escobar H.
}

Pontificia Universidad Católica de Chile, Santiago Departamento de Pediatría Servicio de Pediatría (CSC, MLC)

Sección Infectología (KAV) Sección Neurología (ANF, REH) Hospital Dr. Sótero Del Río, Santiago, Chile Unidad de Neurología Pediátrica (CMS)

Recibido: 31 marzo 2008 Aceptado: 17 septiembre 2008

Correspondencia a: Raúl Escobar H. rescobar@med.puc.cl

\section{Ramsay Hunt syndrome in children: Four cases and review}

Ramsay-Hunt Syndrome (RHS) is a rare affection characterized by peripheral facial paralysis (PFP), skin eruption in the auricular canal and cochleovestibular symptoms. It is produced by varicella-zoster virus (VZV) reactivation at the geniculate ganglia. We report four patients between 3 and 17 years-old with RHS. Earache was the first symptom in two cases and three had cochleovestibular compromise. The direct immunofluorescence from the vesicular lesion was positive for VZV in two of them. All patients received treatment with acyclovir and in three cases, this was associated with steroids. Three children had complete resolution of the PFP. RHS is an infrequent disease in the pediatric population and it should be suspected in children with PFP, erythema, vesicles and/or auricular pain. Early treatment with acyclovir therapy could improve the recovery rate of facial nerve palsy.

Key words: Ramsay Hunt syndrome, peripheral facial paralysis, oticus herpes zoster, varicella zoster. Palabras clave: Síndrome de Ramsay Hunt, parálisis facial periférica, herpes zoster ótico, varicela zoster.

\section{Introducción}

$\mathrm{E}$ 1 síndrome de Ramsay-Hunt (SRH) se produce por la reactivación del VVZ en el ganglio geniculado, originando lesiones vesiculares, radiculoneuropatía y ganglionitis; los pacientes cursan característicamente con parálisis facial periférica (PFP), erupción vesicular en el pabellón auricular y afectación cocleo-vestibular del mismo lado ${ }^{1-4}$. Fue descrito por primera vez por James Ramsay-Hunt en 1907, quien introdujo el término de herpes zoster (HZ) ótico ${ }^{5-7}$.

La mayoría de los reportes pediátricos son descripciones de casos aislados. Las series publicadas reportan casos de población infantil y adulta en sus análisis, siendo infrecuentes los casos de PFP secundarias a VVZ.

Se describe la reactivación del VVZ como causa de PFP en 25 a $50 \%$ de todos los niños con PFP entre 6 y 15 años, y 9 a $10 \%$ en los niños bajo 6 años de edad con PFP; a menor edad de adquisición de la infección por VVZ mayor es el riesgo que ocurra este tipo de reactivación ${ }^{4,7}$.

Las manifestaciones clínicas dependen de la estructura neuronal donde ocurre la reactivación viral ${ }^{8}$.

Comunicamos a continuación la presentación clínica, tratamiento y evolución de cuatro pacientes pediá- tricos con diagnóstico de SRH y se revisa la información publicada al respecto en la literatura médica.

\section{Pacientes y Método}

Estudio descriptivo, retrospectivo. Fueron incluidos aquellos pacientes bajo 18 años de edad con diagnóstico clínico de SRH, caracterizado por PFP y lesiones vesiculares en el pabellón auricular o conducto auditivo externo (CAE), que fueron controlados en el departamento de neurología pediátrica de la Red de Salud UC, durante los años 2006-2007.

De cada paciente se registraron los antecedentes biodemográficos y mórbidos, historia clínica y examen físico, resultados de exámenes de laboratorio, incluyendo neuroimágenes, tratamiento recibido y evolución a seis meses plazo. El grado de PFP se estableció según la clasificación de House-Brackmann (Tabla 1) ${ }^{9}$.

La revisión de la literatura médica se realizó en las bases de datos scielo y PubMed.

\section{Casos clínicos}

Paciente 1: Niña de 3 años, con antecedente de varicela a los siete meses de edad. Consultó por des- 
viación de la comisura labial derecha, fiebre y eritema doloroso en el pabellón auricular ipsilateral. Se le diagnosticó una PFP grado III (Tabla 1), y se indicó tratamiento con corticosteroides y analgésicos. Consultó dos días después por dificultad de la marcha. Al examen físico, la paciente estaba irritable, con una PFP derecha y en el pabellón auricular derecho se apreciaba eritema, con dos lesiones vesiculares bajo el trago. Inicialmente se pesquisó disminución de los reflejos osteotendíneos (ROT) rotuliano y aquiliano derechos, con fuerzas conservadas y sin signos cerebelosos. La alteración de los ROT fue transitoria, hallazgo que no se corroboró durante la evolución del episodio agudo y fue interpretado como secundario a la dificultad en la realización del examen físico inicial, dada la marcada irritabilidad de la paciente. La marcha fue inestable, con lateralización a derecha.

Exámenes de laboratorio: Estudio de hisopado de la base de la lesión vesicular: IFD para VVZ positiva. Resonancia magnética (RM) cerebral: refuerzo anormal de los nervios VII $^{\circ}$ y VIII $^{\circ}$ dentro del conducto auditivo interno, especialmente hacia el fundus, lo que indica inflamación de ambos nervios craneanos. Estudio del LCR: análisis citoquímico normal, tinción de Gram y cultivo corriente negativos. La detección de ADN viral por RPC para VVZ en LCR y saliva fue negativa.

Tratamiento: Prednisona $1 \mathrm{mg} / \mathrm{kg} /$ día durante 5 días y aciclovir (ACV) $80 \mathrm{mg} / \mathrm{kg} /$ día vía oral (vo), en 4 tomas, por 10 días.

Evolución: Durante el seguimiento ambulatorio se le realizó potenciales evocados auditivos de tronco (PEAT), que resultaron compatibles con hipoacusia sensorioneural derecha. El control con audiometría un mes después fue normal.

A los 7 meses de seguimiento se apreciaba mejoría de la PFP (grado I) (Tabla 1).

Paciente 2: Niño de 9 años, sin antecedentes de varicela, que consultó por dos días de cefalea intensa, asimetría facial, eritema y dolor en el pabellón auricular izquierdo. Al examen se constató una PFP izquierda grado IV y vesículas en el CAE del mismo lado.

Exámenes de laboratorio: El estudio del LCR fue compatible con encefalitis viral: 60 células $/ \mathrm{mm}^{3}$ con $75 \%$ de mononucleares. La tinción de Gram y el cultivo corriente no detectaron bacterias. RPC para herpes simplex tipo 1 en LCR negativo. IFD para VVZ de la lesión vesicular fue positiva. La RM cerebral fue normal.

Tratamiento: Debido al compromiso encefálico se trató con ACV endovenoso (ev) $10 \mathrm{mg} / \mathrm{kg}$, cada 8 horas, durante cinco días, pasando a vo para completar 21 días con $20 \mathrm{mg} / \mathrm{kg}$, cada 6 horas. No recibió
Tabla 1. Clasificación de House-Brackmann para parálisis facial periférica

\begin{tabular}{|c|c|}
\hline Grado & Descripción \\
\hline I & Función normal en todas sus áreas \\
\hline II & $\begin{array}{l}\text { Disfunción leve } \\
\text { Global: debilidad superficial notoria sólo a la inspección cercana } \\
\text { Puede haber mínima sincinesias. Al reposo, tono y simetría normal } \\
\text { Movimiento de la frente: función de buena a moderada } \\
\text { Ojo: cierre completo con mínimo esfuerzo } \\
\text { Boca: asimetría mínima al movimiento }\end{array}$ \\
\hline III & $\begin{array}{l}\text { Disfunción leve a moderada } \\
\text { Global: obvia pero no desfigurativa, asimetría al reposo y a la actividad } \\
\text { Existencia de sincinesias y/o aumento del tono de músculos faciales } \\
\text { Movimiento de la frente: moderados a ligeros } \\
\text { Ojo: Cierre completo con esfuerzo } \\
\text { Boca: ligera debilidad con el máximo esfuerzo }\end{array}$ \\
\hline IV & $\begin{array}{l}\text { Disfunción moderada a intensa } \\
\text { Global: debilidad obvia y/o asimetría desfigurativa Al reposo, asimetría } \\
\text { Movimiento de la frente: ninguno } \\
\text { Ojo: cierre incompleto } \\
\text { Boca: asimetría al esfuerzo }\end{array}$ \\
\hline V & $\begin{array}{l}\text { Disfunción intensa } \\
\text { Global: solamente movimientos apenas perceptibles. Asimetría al reposo } \\
\text { Movimiento de la frente: ninguno } \\
\text { Ojo: cierre incompleto } \\
\text { Boca: movimientos ligeros y al movimiento }\end{array}$ \\
\hline VI & $\begin{array}{l}\text { Parálisis total } \\
\text { Ningún movimiento }\end{array}$ \\
\hline
\end{tabular}

corticosteroides ante la sospecha inicial de encefalitis por VHS.

Evolución: Respuesta terapéutica satisfactoria. El seguimiento a seis meses evidenció la desaparición del compromiso facial izquierdo (grado I).

Paciente 3: Niña adolescente de 16 años, sin antecedentes de varicela. Consultó por asimetría facial, otalgia y tinitus a derecha, con 24 horas de evolución, y odinofagia durante dos días. Al examen físico se constató una PFP derecha grado IV, lesiones vesiculares linguales y en el CAE derecho.

Exámenes de laboratorio: Estudio del $\mathrm{VIII}^{\circ}$ nervio craneano mostró lesión vestibular periférica e hipoacusia sensorioneural derecha. No se realizó estudio virológico.

Tratamiento: ACV $800 \mathrm{mg}$ cada 4 horas vo, en cinco tomas, durante 14 días y prednisona $20 \mathrm{mg}$ cada 12 horas por 5 días.

Evolución: Durante la primera semana de evolución desaparecieron la otalgia y el tinitus. Al cuarto mes de seguimiento, un control con PEAT fue normal y hubo desaparición completa de la PFP. 
Figura 1. Paciente con parálisis facial periférica.
Paciente 4: Adolescente varón de 17 años, con antecedente de varicela a los 5 años. Comenzó con otalgia derecha, a lo que se agregó vértigo objetivo a derecha, vómitos y asimetría facial. Al examen se detectó una PFP derecha grado IV, vesículas en el CAE ipsilateral (Figuras 1 y 2) y lateropulsión a derecha, sin nistagmus espontáneo ni posicional.

Exámenes de laboratorio: IFD para VVZ de lesión vesicular fue negativa. Estudio de $\mathrm{VIII}^{\circ}$ nervio craneano mostró una hiper-excitabilidad vestibular leve. La audiometría resultó normal.

Tratamiento: ACV $800 \mathrm{mg}$ cada 4 horas vo, en cinco tomas, durante 14 días y prednisona $20 \mathrm{mg}$ cada 12 horas, por 5 días.

Evolución: Cedió la sintomatología cocleo-vestibular, pero aún presentaba la PFP derecha al mes de seguimiento. Se apoyó con kinesiterapia facial, observándose una buena respuesta. Al cuarto mes de seguimiento persistía una PFP grado II.
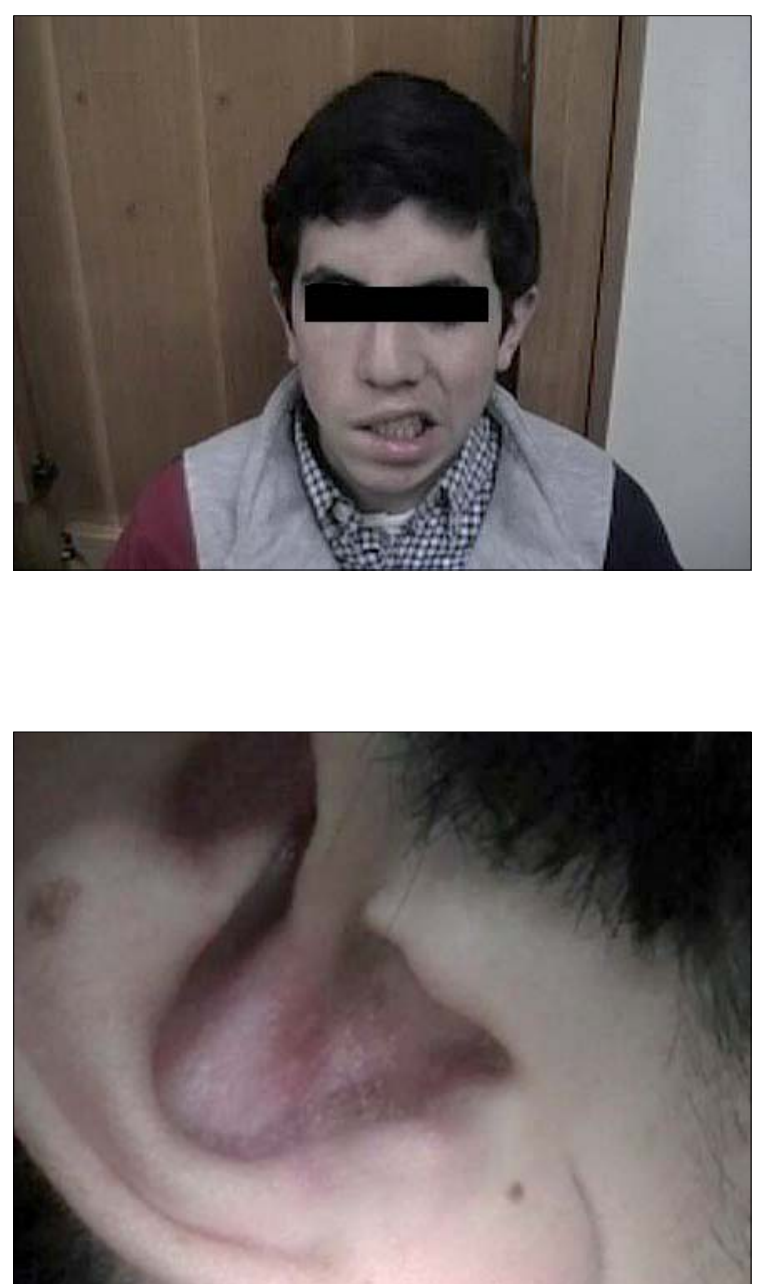

\section{Discusión y revisión de la literatura}

El SRH corresponde a un tipo de reactivación del VVZ, también llamado HZ ótico. Según las series reportadas, representa aproximadamente $7 \%$ del total de las PFP, siendo mucho menos frecuente que la parálisis de Bell o "a frigori" 10 . En los reportes en niños, se describe con mayor frecuencia en aquellos que han sido expuestos al VVZ in útero o antes de los 2 años de vida $^{4,7}$.

Manifestaciones clínicas. Los signos y síntomas clínicos corresponden a:

- Rash eritematoso en el CAE, pabellón auricular, mucosa del paladar y/o la lengua ${ }^{11}$; en aproximadamente $80 \%$ de los casos en edad pediátrica se presenta con vesículas, siendo éstas más frecuentes que en el SRH descrito en adultos. Las lesiones pueden aparecer en forma simultánea con la PFP o hasta en la mitad de los casos, varios días posteriores a ésta ${ }^{11}$.

- Compromiso de nervios craneanos, producto del edema secundario a la reacción inflamatoria provocada por el VVZ, el que ocasiona hipoxia y daño neuronal, pudiendo incluso llegar a la degeneración neuronal. El compromiso del $\mathrm{VII}^{\mathrm{O}}$ nervio craneano determina paresia o PFP del lado ipsilateral a las lesiones en la piel y su intensidad se determina según la clasificación de House-Brackmann (Tabla 1). El compromiso del VIII ${ }^{\circ}$ nervio craneano desencadena hipoacusia sensorio-neural, acúfenos, tinitus, otalgia, vértigo y alteración de la marcha. Los nervios craneanos $\mathrm{V}^{\mathrm{o}}$, IX $\mathrm{IX}^{\mathrm{o}} \mathrm{X}^{\mathrm{o}}$ también pueden verse afectados, ocasionando en estos casos polineuritis. Las posibles complicaciones a largo plazo son: déficit motor facial permanente, ulceración corneal secundaria al lagoftalmo y neuralgia postherpética; esta última es inusual en niños y con pronóstico más favorable que la observada en adul$\operatorname{tos}^{11,12}$.

En los casos expuestos, si bien la presentación clínica fue heterogénea, en todos ellos, la aparición de vesículas, principalmente en el pabellón auricular, permitió hacer el diagnóstico en forma precoz. En tres de los cuatro pacientes, además de la PFP, hubo compromiso del nervio cocleo-vestibular ipsilateral a la parálisis, tanto de su rama coclear en el paciente 3 , como de la vestibular en los pacientes 1 y 4 .

Diagnóstico. Es fundamentalmente clínico; sin embargo, puede ser necesario el apoyo de exámenes de laboratorio, en especial para realizar un diagnóstico diferencial con otras patologías. Se dispone de:
Figura 2. Eritema y vesículas cho. 
- Detección de antígenos virales mediante IFD para VVZ de la lesión vesicular de la piel o mucosa oral. La sensibilidad de esta prueba para vesículas en piel es de 60 a 93\% y la especificidad varía entre 94 y $99 \% 0^{12,13}$

- Cultivo viral de la lesión vesicular: la utilidad clínica del aislamiento viral tradicional está limitada por la lentitud en sus resultados (aproximadamente 28 días). El cultivo acelerado o shell vial disminuye este tiempo en 3 a 5 días. La sensibilidad del aislamiento viral es baja, variando entre 30 y $40 \%$ para el cultivo viral tradicional $^{12}$ y aproximadamente $60 \%$ para el cultivo acelerado; la especificidad de ambos es de $100 \%{ }^{13}$.

- Detección de ADN viral por RPC convencional o en tiempo real: esta técnica logra aumentar la sensibilidad de los cultivos (convencional y shell vial) en 60 y $20 \%$ respectivamente, y sus resultados pueden obtenerse en $24 \mathrm{hrs}^{13,14}$.

En dos de los tres casos en que se realizó IFD de la lesión vesicular se logró confirmar la presencia de VVZ, mostrando la utilidad de este examen en el apoyo etiológico del SRH. Sólo en un paciente se realizó RPC en una muestra de vesícula y saliva, puesto que en nuestro medio sólo recientemente se cuenta con este examen. Se requiere más experiencia con esta técnica para determinar su utilidad clínica. En la serie que reporta Furuta $^{8}$, de 30 pacientes con PFP, sólo dos presentaron diagnóstico clínico de SRH. De esta misma serie, nueve pacientes fueron asociados a VVZ sin presentar lesiones vesiculares, demostrándose su reactivación por medio de RPC para VVZ en saliva, lo que pudiera asimilarse a la entidad descrita como "zoster sin herpes", donde la reactivación del VVZ ocurre con dolor y sin lesiones herpéticas ${ }^{8}$. Frente a esto último, sería interesante el estudio virológico de una muestra de saliva o en exudado de la piel del $\mathrm{CAE}^{15}$ en todo niño con PFP, en búsqueda de "SRH sin herpes", evitando el diagnóstico errado de parálisis de Bell.

- Análisis de LCR: el citoquímico, cultivo y estudio viral de LCR pueden ser de utilidad para confirmar la co-existencia de meningitis viral, como ocurrió con el paciente 2, o cuando hay necesidad de establecer un diagnóstico diferencial (paciente 1), en especial en niños con trastorno de la marcha, cefalea, y particularmente, cuando no hay lesiones vesiculares concomitantes.

- Neuroimágenes: La RM de cerebro puede ser de gran utilidad en el diagnóstico diferencial con tumores cerebrales si hay trastorno de la marcha o síndrome de Guillain Barré, variedad Müller Fisher, como ocurrió con la paciente 1, en quien se planteó el diagnóstico diferencial por el hallazgo inicial de ROT ausentes e inestabilidad de la marcha. Ade- más, puede ser de utilidad cuando se confirma el compromiso de ganglios de nervios craneanos, lo que se logra con la RM con gadolinio, que muestra un intenso realce en la zona del ganglio geniculado, CAE, mastoides, oído medio, oído interno, nervio facial y nervio cocleo-vestibular ${ }^{16-18}$.

- Estudio de audición: Los PEAT, audiometría y la prueba calórica son útiles para la certificación de compromiso de $\mathrm{VIII}^{\circ}$ nervio craneano, al momento del debut del SRH y son fundamentales en el seguimiento cuando hay compromiso del nervio cocleovestibular. La elección de cada examen dependerá de la edad del paciente, su capacidad de cooperación para realizar cada uno de ellos y de la rama del VIII $^{\circ}$ nervio craneano que se vea afectada.

Tratamiento. El uso de corticosteroides y antivirales, su duración y vía de administración ha sido muy controversial debido a las escasos estudios en pacientes pediátricos.

El diagnóstico precoz del SRH es importante ya que el tratamiento antiviral precoz pudiera influir en la resolución de los síntomas. El tratamiento estándar de primera línea para el $\mathrm{HZ}$, es $\mathrm{ACV}$, ya sea por vía ev o $v^{19}$. La terapia antiviral es eficaz contra la replicación del VVZ y puede impedir una mayor proliferación y propagación de virus; sin embargo, no lo erradica. La evidencia disponible, sugiere que el tratamiento antiviral oral puede disminuir la intensidad del HZ, reducir el tiempo de diseminación del virus y la formación de nuevas lesiones, acelerando la curación y reduciendo el tiempo de dolor ${ }^{20}$. En general, se sostiene que el uso de antivirales orales se limita a un período de siete a diez días y debe iniciarse dentro de las 72 horas de la aparición de la erupción. A pesar de lo anterior, los antivirales no evitarían el dolor en el período agudo ni post-herpético ${ }^{20}$. Se encuentra en proceso de protocolo la revisión sistemática sobre terapia antiviral para SRH en adultos ${ }^{20}$.

En relación a la asociación de terapia corticoesteroidal, Kinishi ${ }^{11}$ reunió 138 pacientes con SRH, 91 tratados con ACV y corticosteroides y 47 sólo con corticosteroides; en este último grupo el tratamiento fue efectuado con metilprednisolona ev. Ambos grupos no tenían diferencias estadísticamente significativas en el grado de PFP pre-tratamiento; sin embargo, en la evaluación post tratamiento, en el grupo tratado con corticosteroides más $\mathrm{ACV}$, el $90 \%$ presentó recuperación completa, en comparación con aquellos tratados sólo con corticosteroides, grupo en que sólo $64 \%$ experimentó recuperación completa. Este hallazgo sugiere que la asociación de un potente anti-inflamatorio y un antiviral que acorte la replicación viral, previene la degeneración de la función nerviosa. 
El estudio de Ramos $^{21}$, asignó en forma aleatoria 45 pacientes adultos con PFP a terapia con corticosteroides solamente, o a corticoterapia más ACV. Aquellos con PFP de Bell recibieron corticosteroides vo y a los con diagnóstico de SRH se les administró corticosteroides ev. En los 15 pacientes con SRH que utilizaron asociación de corticosteroides y ACV hubo un mejor pronóstico en la recuperación de su parálisis, no así en aquellos con parálisis de Bell.

Adicionalmente, $\mathrm{Ko}^{22}$, reunió 30 pacientes que recibieron tratamiento con ACV ev y prednisona. Sus resultados sugieren que la mejoría de la PFP dependía de tres variables: edad de presentación, polineuritis asociada y grado inicial de la PFP. La recuperación con el tratamiento bi-asociado fue favorable y la posibilidad de un buen resultado era mayor cuando el grado inicial de parálisis fue alto. Resultados negativos se observaron en relación a mayor edad de presentación y cuando hubo polineuritis asociada a la PFP.

La terapia con corticosteroides, es utilizada, a menudo, con el objeto de reducir la inflamación del nervio y el dolor asociados ${ }^{23}$. En el caso del SRH, la indicación sería de utilidad en pacientes con dolor moderado a intenso o cuando se asocia a polineuritis; en este último caso, con el objeto de mejorar los resultados motores, además de reducir el daño en los nervios craneanos por compresión. Pese a todo ello, el beneficio de dicho tratamiento no ha sido sistemáticamente estudiado y pudiera utilizarse como terapia complementaria a la terapia antiviral ${ }^{19,20}$.

En nuestros pacientes se prefirió usar esquema biasociado con ACV por un mínimo de 10 días y prednisona durante cinco días, a excepción del paciente 2 en el cual no se empleó tratamiento corticoesteroidal por la sospecha inicial de encefalitis herpética, y se prolongó el tratamiento antiviral por 21 días. La evolución de la PFP fue satisfactoria en la mayoría de los casos, todos mejoraron el grado de PFP y tres de ellos se recuperaron completamente, a seis meses de seguimiento.

Con respecto a tratamientos complementarios, la oclusión ocular y el uso de lágrimas artificiales, mientras no haya recuperación del cierre palpebral, son esenciales para evitar complicaciones oculares. La kinesiterapia facial se ha recomendado para casos con mala respuesta a tratamiento medicamentoso, PFP grados IV a VI de House-Brackmann y no antes de cuatro a ocho semanas de evolución, pues antes debe considerarse la recuperación espontánea. El tratamiento kinésico considera aspectos básicos de re-educación muscular supervisada y uso de electroterapia en casos seleccionados, por lo que se sugiere el juicio clínico para la aplicación de este recurso en músculos paralizados o con mínima contracción visible o palpable, por punto motor y no en masa, para mantener el trofismo muscular. La re-educación muscular frente al espejo ("mime therapy") es la piedra angular del tratamiento neuro-rehabilitador, no ha demostrado ser beneficiosa ni deletérea en pacientes con parálisis de Bell ${ }^{23-26}$.

Seguimiento: Se aconseja el seguimiento objetivando el compromiso del $\mathrm{VIII}^{\circ}$ nervio craneano y su control periódico para verificar la mejoría. El seguimiento recomendado es con audiometría y/o prueba funcional del VIII $^{\circ}$ nervio craneano; en pacientes que no cooperan son de utilidad los PEAT ${ }^{25-30}$.

En tres de los casos presentados se realizó audiometría, la que detectó hipoacusia sensorioneural derecha en el paciente 3 . En el paciente 1 además se realizó PEAT al inicio del cuadro, con resultado anormal, mientras que en el seguimiento la audiometría no mostró alteraciones. Se realizó además prueba de $\mathrm{VIII}^{\mathrm{o}}$ nervio craneano completo a dos pacientes. En el caso del paciente 3 fue motivado por sus síntomas cocleares, destacando una lesión vestibular periférica; se le realizó un control posterior con PEAT para verificar mejoría de la sintomatología coclear, el cual resultó normal. En el paciente 4 el motivo del examen fue la importante sintomatología vestibular, mostrando sólo hiper-excitabilidad vestibular leve, en la prueba calórica, sin otro compromiso. No requirió control posterior por desaparición de los síntomas. En tres de nuestros pacientes hubo una muy buena recuperación; en el cuarto paciente persistió la PFP por lo que recibió apoyo kinésico, con buena evolución posterior.

Prevención: La vacuna con VVZ vivo atenuado fue fabricada en Japón en 1974 y aprobado su uso en E.U.A. en 1995. Ha tenido un efecto profundo en la epidemiología de la varicela y también del HZ, ya que retrasa y evita la infección primaria por VVZ y con ello también retrasa la aparición del $\mathrm{HZ}^{32}$. La incidencia de $\mathrm{HZ}$ en los vacunados contra varicela es de 14/100.000 bajo 19 años de edad versus 20-63/100.000 en aquellos que adquirieron la infección nativa ${ }^{32}$. Niños con leucemia linfoblástica aguda, vacunados contra varicela, desarrollaron $\mathrm{HZ}$ en 0,8/1.000; en cambio, aquellos con adquisición de la infección natural, la incidencia de $\mathrm{HZ}$ se elevó a 2,46/1.000 31 .

Sólo en dos de nuestros pacientes se encontró el antecedente de varicela y ninguno había sido vacunado, lo que pudiera deberse a que, en ocasiones, la varicela puede no ser percibida por los padres por cursar en forma extremadamente atenuada; la ausencia de antecedente de varicela no descarta, entonces, que el SRH pueda deberse a una reactivación del VVZ.

Los antecedentes entregados confirman la importancia de la vacunación de niños sanos en la prevención de la varicela y de sus complicaciones inmediatas y tardías. Desgraciadamente, en hospederos inmuno- 
comprometidos quienes están expuestos a un mayor riesgo de adquirir una infección por varicela complicada, a tener una enfermedad más prolongada y a desarrollar $\mathrm{HZ}$ en sitios inusuales, incluso con diseminación visceral ${ }^{32}$, está contraindicada esta vacunación.

SRH e inmunocomprometidos: Esta patología ha sido descrita en pacientes inmunocomprometidos, principalmente aquellos con infección por VIH/SIDA, como reportes de casos únicos ${ }^{33-35}$. No se recomienda, de rutina, evaluar una posible inmunodeficiencia en un paciente que curse con SRH; tampoco es recomendado de rutina el estudio de infección por VIH en los niños o adolescentes que presentan HZ. Probablemente, lo más importante será evaluar, paciente a paciente, si hay algún otro factor que oriente a la sospecha de inmunodeficiencia para proceder al estudio de su inmunidad.

En suma, el SRH es una entidad poco frecuente en pediatría, que debe sospecharse en niños con una PFP, eritema, vesículas y/o dolor auricular. Es recomendable la confirmación etiológica del VVZ, por medio de IFD o aislamiento viral a partir de las vesículas $\mathrm{y}$, en ausencia de ellas, su búsqueda en saliva o secreción del CAE, por medio de RPC para ADN viral. El estudio complementario con neuro-imágenes es útil cuando se han planteado otros diagnósticos diferenciales. El tratamiento precoz, asociado, de ACV con corticosteroides, podría mejorar la evolución de la PFP, recomendación extrapolada de la experiencia en pacientes adultos; sin embargo, no existen estudios de buena calidad metodológica que demuestren su beneficio. Son necesarios estudios multicéntricos y rando- mizados para encontrar el mejor esquema de tratamiento en los pacientes pediátricos. El seguimiento clínico es importante complementarlo con evaluación del VIII ${ }^{\circ}$ nervio craneano. El apoyo con kinesiterapia motora facial está indicado si persiste la PFP después de 4 a 8 semanas de evolución.

Es de vital importancia considerar que la vacuna anti-varicela es recomendable para disminuir el riesgo de este tipo de complicaciones.

\section{Resumen}

El síndrome de Ramsay-Hunt (SRH) corresponde a una inusual afección caracterizada por parálisis facial periférica (PFP), erupción en el pabellón auricular ipsilateral y compromiso cocleo-vestibular. Es producida por reactivación del virus varicela zoster (VVZ) a nivel del ganglio geniculado. Se reporta una serie de cuatro pacientes entre 3 y 17 años de edad con SRH. La otalgia fue el primer síntoma en dos casos, tres de ellos presentaron sintomatología vestibular periférica y uno déficit cócleo-vestibular. La inmunofluorescencia directa de hisopado de lesión vesicular fue positiva para VVZ en dos niños. Todos recibieron tratamiento con aciclovir y tres recibieron además corticoesteroides. Tres niños tuvieron recuperación clínica completa. El SRH es una entidad poco frecuente en pediatría y debe sospecharse en niños con PFP, eritema, vesículas y/o dolor auricular, ya que el tratamiento precoz con aciclovir pudiera mejorar la evolución de la PFP.

\section{Referencias}

1.- Hato N, Kisaki H, Honda N, Gyo K, Murakami S, Yanagihara N. Ramsay Hunt syndrome in children. Ann Neurol 2000; 48: 254-6.

2.- Van de Steene V, Kuhweide R, Vlaminck S, Casselman J. Varicella zoster virus: Beyond facial paralysis. Acta Otorhinolaryngol Belg 2004; 58: 61-6.

3.- Kuhweide R, Van de Steene V, Vlaminck S, Casselman J. Ramsay Hunt syndrome: Pathophysiology of cochleovestibular symptoms. J Laryngol Otol 2002; 116: 844-8.

4.- Alcalá P, Zubiaur A, Ronda J M, Herrero A, López D, Flores J. Síndrome de Ramsay-Hunt. An Esp Pediatr 2002; 56: 269-70.

5.- Kimitsuki T, Komiyama S. Ramsay-Hunt syndrome in a 4-year-old child. Eur Arch Otorhinolaryngol 1999; 256 (suppl 1):
S6-S7.

6.- Koga C, Iwamoto O, Aoki M, Nakamura C, Kusukawa J, Matsuishi T. Ramsay-Hunt syndrome with vesicular stomatitis in a 4-year-old Infant. Oral Surg Oral Med Oral Pathol Oral Radiol Endod 2006; 102: e37-e9.

7.- Balatsouras D G, Rallis E, Homsioglou E, Fiska A, Korres S G. Ramsay Hunt syndrome in a 3-month-old infant. Pediatr Dermatol 2007; 24 (1): 34-7.

8. - Furuta Y, Ohtani F, Aizawa H, Fukuda S, Kawabata H, Bergström T. Varicella-zoster virus reactivation is an important cause of acute peripheral facial paralysis in children. Pediatr Infect Dis J 2005; 24 (2): 97-101.

9.- Meadows A, Hall N, Shah-Desai S, Low J L, Manners R. The House-Brackmann system and assessment of corneal risk in facial nerve palsy. Eye 2000; 14: 353-7.

10.- Pino V, Marqués L, Pereda J M, Montero C, Blasco A. Síndrome de Ramsay-Hunt.
Nuestra experiencia y revisión de la literatura. ORL-DIPS 2002; 29: 120-3.

11.- Kinishi M, Amatsu M, Mohri M, Saito M, Hasegawa T, Hasegawa S. Acyclovir improves recovery rate of facial nerve palsy in Ramsay Hunt syndrome. Auris Nasus Larynx 2001; 28: 223-6.

12.- Coffin S E, Hodinka R L. Utility of direct immunofluorescence and virus culture for detection of varicella-zoster virus in skin lesions. J Clin Microbiol 1995; 33: 2792-5.

13.- Chan R, Brandt K, Horsman G. Comparison of chemicon simulfluor direct fluorescent antibody staining with cell culture and shell vial direct immunoperoxidase staining for detection of herpes simplex virus and with cytospin direct immunofluorescence staining for detection of varicella-zoster virus. Clin Diag Lab Immunol 2000; 8 (5): 909-12.

14.- Espy M, Teo R, Ross T, Svien K, Wold A, Uhl J, et al. Diagnosis of varicella-zoster virus infections in the clinical laboratory by 
light cycler PCR. J Clin Microbiol 2000; 38: 3187-9.

15.- Murakami S, Miyamoto N, Watanabe N, Matsuda F. Alpha herpes virus and facial palsy. Nippon Rinsho 2000; 58: 906-11.

16.- Bravo D, González C, Foglia F, Ramírez R, Amilibia C, Dicenta S. Manifestaciones otorrinolaringológicas del virus varicelazoster. Acta Otorrinolaringol Esp 1999; 50: 225-7.

17.- Nogueira R, Seeley W. Ramsay Hunt syndrome associated with spinal trigeminal nucleus and tract involvement on MRI. Neurology 2003; 61: 1306-7.

18.- Grose C, Bonthius D, Afifi A K. Chickenpox and the geniculate ganglion: Facial nerve palsy, Ramsay Hunt syndrome and acyclovir treatment. Pediatr Infect Dis J 2002; 21: 615-7.

19. - Dworkin R H, Johnson R W, Breuer J, Gnann J W, Levin M J, Backonja M, et al. Recommendations for the management of herpes zoster. Clin Infect Dis 2007; 44 Suppl 1: S1-26.

20.- Uscategui T, Doree C, Chamberlain I J, Burton M J. Antiviral therapy for Ramsay Hunt syndrome (herpes zoster oticus with facial palsy) in adults. Cochrane Database of Systematic Reviews, issue 3, 2008.

21.- Ramos Macías A, de Miguel Martínez I, Martín Sánchez A M, Gómez González J L,
Martín Galán A. The incorporation of acyclovir into the treatment of peripheral paralysis. A study of 45 cases. Acta Otorrinolaringol Esp 1992; 43: 117-20.

22.- Ko J Y, Sheen T S, Hsu M M. Herpes zoster oticus treated with acyclovir and prednisolone: Clinical manifestations and analysis of prognostic factors. Clin Otolaryngol Allied Sci 2000; 25: 139-42.

23. - Pérez C E, Gámez M C, Guzmán G J M, Escobar R D, López R V M, Montes de O R $\mathrm{D}$, et al. Guía clínica para la rehabilitación del paciente con parálisis facial periférica. Rev Med IMSS 2004; 42: 425-36.

24.- Beurskens C H, Heymans P G. Physiotherapy in patients with facial nerve paresis: Description of outcomes. Am J Otolaryngol 2004; 25: 394-400.

25.- Ohtake P J, Zafron M L, Poranki L G, Fish D R. Does electrical stimulation improve motor recovery in patients with idiopathic facial (Bell) palsy?. Phys Ther 2006; 86: 1558-64.

26. - Teixeira L J, Soares B G, Vieira V P, Prado G F. Physical therapy for Bell s palsy (idiopathic facial paralysis). Cochrane Database Syst Rev 2008; 3: CD006283.

27.- Sweeney C J, Gilden D H. Ramsay Hunt syndrome. J Neurol Neurosurg Psychiatry 2001; 71: 149-54.

28.- Ohtani F, Furuta Y, Aizawa H, Fukuda S.
Varicella-zoster virus load and cochleovestibular symptoms in Ramsay Hunt syndrome. Ann Otol Rhinol Laryngol 2006; 115: 233-8.

29.- Walther L E, Prosowsky K, Walther A, Gudziol H. Herpes zoster oticus: Symptom constellation and serological diagnosis. Laryngorhinootologie. 2004; 83: 355-62.

30.- Lu Y C, Young Y H. Vertigo from herpes zoster oticus: superior or inferior vestibular nerve origin?. Laryngoscope 2003; 113 : 307-11.

31.- Vázquez M, Shapiro E D. Varicella vaccine and infection with varicella-zoster virus. $\mathrm{N}$ Engl J Med 2005; 352: 439-40.

32.- Leung A K, Robson W L, Leong A G. Herpes zoster in childhood. J Pediatr Health Care. 2006; 20: 300-3

33.- Pazos-Añón R, Machado-Costa C, Farto E Abreu J. Ramsay-Hunt syndrome complicated with cerebral venous thrombosis in an HIV-1-infected patient. Enferm Infecc Microbiol Clin 2007; 25: 69-70.

34.- Sobn A J, Tranmer P A. Ramsay Hunt syndrome in a patient with human immunodeficiency virus infection. J Am Board Fam Pract 2001; 14: 392-4.

35.- Malik Z A, Litman N. A 17-year-old boy with facial palsy and hyperacusis. Pediatr Infect Dis J 2008; 27: 646. 